\title{
New perspectives in the drug management of allergy and respiratory diseases
}

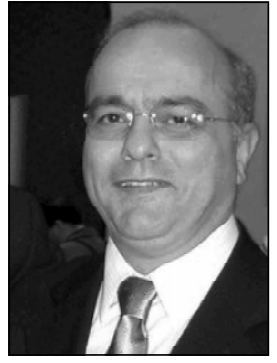

Dirceu Solé ${ }^{1}$

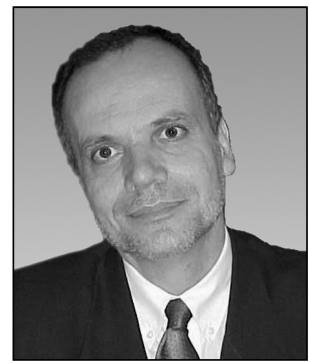

Sérgio L. Amantéa²
In defining the focus of the present Jornal de Pediatria supplement, we tried to group treatment approaches to problems that are commonly observed in routine pediatric practice. Recent advances in the pharmacological context have led to changes in the treatment of many diseases and prompted the need for constant updates regarding the drug arsenal made available for the management of such conditions.

Pediatric specialties such as pulmonology and allergy are linked by scope. They share the same clinical approach in a number of diseases that fall within the domain of both specialties. They also share the same treatment arsenal and the challenge of keeping up to date with specific pharmacological information.

New drugs resulting from advances in clinical research no doubt deserve a dedicated supplement providing updated treatment information on diseases that are common to both pulmonology and allergy. Furthermore, this update should transcend the scope of specialties to encompass general pediatrics.

One of the most important aspects related to clinical pharmacology is the difficulty in establishing treatment boundaries. As the use of certain drugs becomes more frequent, their prescription range is constantly modified. It is common to see "new uses" for "old drugs," with broader application patterns resulting from a favorable safety profile and the pressure to produce results. The constant updates in clinical pharmacology are a common challenge to all of those working with patients.

In defining a scope of action that emphasizes common clinical situations in routine pediatric practice, we are at the same time widening the domain of specialties.

1. Professor titular e livre-docente, Disciplina de Alergia, Imunologia Clínica e Reumatologia, Departamento de Pediatria, Universidade Federal de São Paulo Escola Paulista de Medicina (UNIFESP-EPM), São Paulo, SP, Brasil.

2. Professor adjunto, Departamento de Pediatria, Fundação Faculdade Federal de Ciências Médicas de Porto Alegre (FFFCMPA), Porto Alegre, RS, Brasil. Chefe, Serviço de Emergência Pediátrica, Hospital da Criança Santo Antônio, Complexo Hospitalar Santa Casa, Porto Alegre, RS, Brasil.

Suggested citation: Solé D, Amantéa SL. New perspectives in the drug management of allergy and respiratory diseases. J Pediatr (Rio J). 2006;82(5 Suppl):S125-6. 
In this supplement, the pharmacological approach to diseases such as bronchial asthma and allergic rhinitis is presented in more than one article, with in-depth coverage of the specific use of several drugs (monoclonal anti-IgE antibodies, inhaled corticosteroids, antileukotrienes and the new antihistamines). Unique and particular aspects of anti-infection treatment are discussed, such as antimicrobial resistance and drug allergies. Situations that are commonly faced in clinical practice, such as childhood dermatitis and gastroesophageal reflux disease, are presented from a practical treatment perspective. In addition, controversial issues such as the real benefits of treatment with pre and probiotics, nonsteroidal anti-inflammatory drugs (selective $\mathrm{COX}_{2}$ inhibitors) and drugs for treatment of pediatric pulmonary hypertension add to our challenge.

We hope that our objectives have been met, and also that the convictions and controversies presented herein will serve as stimulus for the search for further pharmacological knowledge concerning common challenges in pediatric practice. 